\title{
Measured foliations and handlebodies
}

\author{
HOWARD MASUR \\ Department of Mathematics, University of Illinois, Chicago, IL 60680, USA \\ (Received 20 February 1984, revised 5 March 1985 and 7 June 1985)
}

\begin{abstract}
We consider the action of the subgroup of the mapping class group consisting of homeomorphisms that extend to the handlebody on Thurston's sphere of measured foliations. Properties of the limit set and domain of discontinuity are described and for genus two it is shown the limit set has measure zero.
\end{abstract}

0. In this paper we initiate a study of the action of the mapping class group of a handlebody on Thurston's sphere of foliations. The action is very different from that of the full mapping class group. It is a fundamental result of Thurston's that the simple closed curves are dense in the sphere of foliations. From that it follows [1] that the mapping class group acts minimally and even ergodically [6]. For the subgroup of diffeomorphisms that extend to the handlebody there is a domain of discontinuity and a nowhere dense limit set. The latter consists of foliations approximated by simple curves on the surface bounding discs in the handlebody.

In the first section we consider properties of this set and the domain of discontinuity, in particular geometric conditions on a foliation which determine which set it lies in. In the second part we consider the special case of genus two where much more can be said. It turns out that the limit set is a kind of Sierpinski set derived by successively removing canonically defined open sets, each a group image of a fixed set. By placing the problem in the context of train tracks we associate to each minimal foliation in the limit set a simplicial process in the sense of Kerckhoff [4] and by directly applying his results on simplicial processes show the limit set has measure zero.

This study had its genesis in Thurston's manuscript [8]. A Heegaard decomposition of genus $g$ of a 3-manifold is determined by a system of $g$ curves and thus a point in the sphere of foliations. Thurston asked whether the closure of the set of points determining a given manifold has measure zero. Since equivalent points under the handlebody group determine homeomorphic manifolds, Thurston's question is related to the measure of the limit set. A related question is which Heegaard decompositions give hyperbolic manifolds. Floyd considered this problem in [2] and has given the answer in terms of the limit set. 
The handlebody group also arises in the study of Schottky groups. Maskit [5] proved that the space of marked Schottky groups of genus $g$ is the quotient of the Teichmuller space of genus $g$ by the subgroup of the handlebody group consisting of homeomorphisms inducing the identity on $\pi_{1}$. Since the sphere of foliations is a natural boundary for Teichmuller space, the action of this subgroup on the sphere serves as a model for deformations at infinity of Schottky groups.

This paper is organized as follows. We assume the reader is familiar with the basic theory of measured foliations found in [1]. $\S 1$ is a discussion of simple curves and is the foundation for the discussion of the domain of discontinuity and limit set found in $\S \S 2,3$. In $\S 4$ we consider the case of genus two and in $\S 5$ prove the measure is zero in that case. This last section contains a review of train tracks and the machinery needed to apply the results of [4].

I would like to thank William Thurston, Nat Kuhn, and William Veech for many helpful suggestions and Steve Kerckhoff for suggesting that the use of train tracks would greatly simplify the measure zero proof given in an earlier version. I would also like to thank the Institute for Advanced Study for their hospitality and support, and the Sloan Foundation for their support during the time this research was conducted.

This research was supported in part by NSF.

1. We begin with notation:

$H_{g}$ is a handlebody of genus $g \geq 2$ with boundary;

$M$ a $C^{\infty}$ surface of genus $g$;

$\mathscr{M F}$ is the space of equivalence classes of measured foliations on $M$;

$\mathscr{P} \mathscr{F}$ is the space of projective measured foliations homeomorphic to $S^{6 g-7}$;

$S$ are the isotopy classes of simple closed curves on $M$;

$S_{t} \subset S$ are the classes of curves that bound discs in $H_{g}$;

$m$ is the natural measure class on $\mathscr{P} \mathscr{F}$.

We refer to curves in $S_{t}$ as $H_{g}$-trivial. Mod $(g)$ is the mapping class group of genus $g, \operatorname{Mod}\left(H_{g}\right)$ is the subgroup of $\operatorname{Mod}(g)$ of the homeomorphisms that extend to homeomorphisms of $H_{g}$. $\operatorname{Mod}_{0}\left(H_{g}\right)$ is the subgroup of Mod $\left(H_{g}\right)$ of homeomorphisms that induce the identity on $\pi_{1}\left(H_{g}\right)$. A disjoint system $\bar{\alpha}=\left(\alpha_{1}, \ldots, \alpha_{g}\right)$ of curves in $S_{t}$ is admissible if they cut $M$ into a sphere with $2 g$ holes. Recall also the basic notion of the intersection number. For $\gamma_{1}, \gamma_{2} \in S, i\left(\gamma_{1}, \gamma_{2}\right)$ denotes the geometric intersection, the minimal number of intersections in the isotopy classes. One defines for $r \in \mathbb{R}_{+}, i\left(r \gamma_{1}, \gamma_{2}\right)=r i\left(\gamma_{1}, \gamma_{2}\right)$. Now for any $F \in \mathcal{M} \mathscr{F}, i(F, \gamma)$ is the minimal transverse length with respect to the measure of $F$ of any $\gamma$ in the isotopy class. This generalizes the geometric intersection.

THEOREM A [7]. $i(F, r \gamma)=r i(F, \gamma)$ extends to a continuous function $i(\cdot, \cdot)$ on $M \mathscr{F} \times$ $\mathcal{M} \mathscr{F}$ which is linear in each factor and $i(f(F), f(G))=i(F, G)$ for all $F, G$ and $f \in \operatorname{Mod}(g)$. If $F$ has no saddle connections ( $F$ arational) and $i(F, G)=0$, then $G$ is topologically conjugate to $F$. 
Lemma 1.1. Suppose $\beta \in S$. Then $\beta \in S_{\mathrm{t}}$ if and only if for any admissible $\bar{\alpha}$ either:

(i) $i\left(\beta, \alpha_{i}\right)=0$ all $i$; or

(ii) $i\left(\beta, \alpha_{i}\right) \neq 0$ some $i$, and for some $\alpha_{j}, \beta$ successively intersects $\alpha_{j}$ in opposite directions. (There are no intersections with any other $\alpha_{k}$ in between these two.)

Proof. Suppose first $\beta \in S_{t}$ and $i\left(\beta, \alpha_{i}\right) \neq 0$ some $i$. Choose $\beta_{1}, \ldots, \beta_{g} \in S$ with $i\left(\beta_{j}, \alpha_{i}\right)=\delta_{i j}$ and $\beta_{j}$ free generators for $\pi_{1}\left(H_{g}\right)$. As a word in $\beta_{j}$ and $\beta_{j}^{-1}, \beta$ is trivial since $\beta \in S_{\text {r }}$. Allowing for cyclic permutations, there must be a juxtaposition $\beta_{j} \beta_{j}^{-1}$ or $\beta_{j}^{-1} \beta_{j}$ somewhere in the word. But this means precisely that $\beta$ crosses $\alpha_{j}$ and then next crosses $\alpha_{j}$ in the opposite direction before crossing any $\alpha_{i \text {. }}$

For the sufficiency suppose for some $\bar{\alpha}$ that $i\left(\beta, \alpha_{i}\right)=0$ all $\alpha_{i}$. The discs bounded by the $\alpha_{i}$ cut $H_{g}$ into a 3-ball. Since $i\left(\beta, \alpha_{i}\right)=0, \beta$ is a simple curve on the bounding sphere which thus bounds a disc in the 3-ball. The proof then proceeds by induction on $i(\bar{\alpha}, \beta)=\sum_{i=1}^{g} i\left(\beta, \alpha_{i}\right)$. Suppose condition (ii) is satisfied for some $\alpha_{j}$. In figure 1 consider the two curves $\alpha_{j}^{\prime}$ and $\alpha_{j}^{\prime \prime}$.

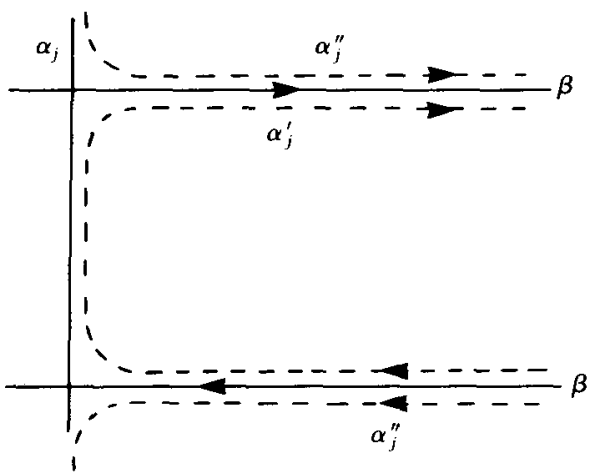

FIGURE 1

Since the two indicated intersections of $\beta$ with $\alpha_{j}$ are successive, $\alpha_{j}^{\prime}$ and $\alpha_{j}^{\prime \prime}$ are disjoint from all other $\alpha_{i}$; hence they are in $S_{t}$. Moreover, neither is homotopic to $\alpha_{j}$ since $i\left(\beta, \alpha_{j}^{\prime}\right)<i\left(\beta, \alpha_{j}\right)$ and $i\left(\beta, \alpha_{j}^{\prime \prime}\right)<i\left(\beta, \alpha_{j}\right)$. Since $\bar{\alpha}$ cuts $M$ into a sphere with $2 g$ holes, exactly one of the two curves $\alpha_{j}^{\prime}$ or $\alpha_{j}^{\prime \prime}$, say $\alpha_{j}^{\prime}$, will separate the two sides of $\alpha_{j}$ in the sphere.

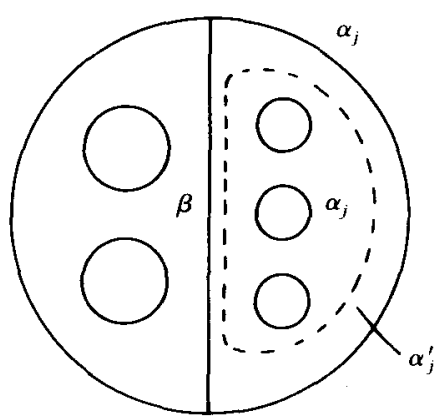

FIGURE 2 
The new system of curves $\bar{\alpha}^{\prime}=\left(\alpha_{1}, \ldots \alpha_{j}^{\prime}, \ldots \alpha_{g}\right)$ with $\alpha_{j}^{\prime}$ replacing $\alpha_{j}$ is admissible. If not, then the curves are dependent in homology. Since $\left(\alpha_{1}, \ldots \alpha_{g}\right)$ is admissible, they are independent. But $\alpha_{j}^{\prime}$ was chosen so that $\alpha_{j}^{\prime}, \alpha_{j}$ and some of the $\alpha_{i}$ bound. Thus $\alpha_{j}$ is a linear combination of $\alpha_{j}^{\prime}$, and the other $\alpha_{i}$ and thus a linear combination of just the $\alpha_{i}$. This is a contradiction. But now $i\left(\bar{\alpha}^{\prime}, \beta\right)<i(\bar{\alpha}, \beta)$, completing the induction step.

THEOREM 1.2. Let $L$ be the closure of $S_{t}$ in $\mathscr{P} \mathscr{F}$. Then $L$ is the unique limit set for both the action of $\operatorname{Mod}\left(H_{g}\right)$ and $\operatorname{Mod}_{0}\left(H_{g}\right)$ on $\mathscr{P} \mathscr{F}$. It is connected and has empty interior.

Remark. By limit set we mean closed, invariant, minimal set.

Proof. $L$ is obviously invariant since $S_{t}$ is invariant under both groups. We show $L$ is minimal for $\operatorname{Mod}_{0}\left(H_{g}\right)$. Let $F, G \in L$ where $G=\lim _{n \rightarrow \infty} \gamma_{n}, \gamma_{n} \in S_{t}$, convergence in the topology of $\mathscr{P P} \mathscr{F}$. Let $U$ be a neighbourhood of $G$ in $\mathscr{P} \mathscr{F}$. If $i\left(F, \gamma_{n}\right) \neq 0$ for some $\gamma_{n} \in U$ then $\lim _{j \rightarrow \infty} \tau_{\gamma_{n}}^{j}(F)=\gamma_{n}$ where $\tau_{\gamma_{n}}$ is the Dehn twist about $\gamma_{n}$. Then $\tau_{\gamma_{n}}^{j}(F) \in U$ for large $j$ and $\tau_{\gamma_{n}} \in \operatorname{Mod}_{0}\left(H_{g}\right)$. If $i\left(F, \gamma_{n}\right)=0$ all large $n$, find $\beta \in S_{t}$ so that $i(F, \beta) \neq 0$ and $i\left(\beta, \gamma_{n}\right) \neq 0$ some $\gamma_{n} \in U$. Then $\beta_{n, k}=\tau_{\gamma_{n}}^{k}(\beta) \in U$ for large $k$ and $i\left(F, \beta_{n, k}\right)=i(F, \beta) \neq 0$. Thus $\tau_{\beta_{n, k}}^{l}(F) \in U$ for large $l$. This proves the $\operatorname{Mod}_{0}\left(H_{g}\right)$ orbit of $F$ enters every neighbourhood of $G$, so the groups act minimally. $L$ is clearly unique since any limit set must contain $S_{t}$.

To show $L$ is connected it is enough to show any two points in $S_{t}$ can be connected by a path in $L$. Suppose then $\alpha_{1}, \alpha_{2} \in S_{t}$. The proof is by induction on $i\left(\alpha_{1}, \alpha_{2}\right)$ and follows the ideas in lemma 1.1. If $i\left(\alpha_{1}, \alpha_{2}\right)=0$ then the path of foliations is $F_{s}$ where $F_{s}$ has closed leaves in the homotopy classes of $\alpha_{1}$ and $\alpha_{2}$ with weights $1-s$ and $s$ respectively. That is: for any $\beta \in S$,

$$
i\left(F_{s}, \beta\right)=(1-s) i\left(\beta, \alpha_{1}\right)+s i\left(\beta, \alpha_{2}\right)
$$

To show $F_{s} \in L$ let $\beta \in S$ be a curve satisfying $i\left(\beta, \alpha_{1}\right)=i\left(\beta, \alpha_{2}\right)=1$. Choose a sequence $\left(n_{1}, n_{2}\right) \in \mathbb{Z}_{+} \times \mathbb{Z}_{+}$with $\lim _{n \rightarrow \infty} n_{1} / n_{2}=(1-s) / s$. The map $f_{n}=\tau_{\alpha_{1}}^{n_{1} \circ} \tau_{\alpha_{2}}^{n_{2}}$ satisfies $\lim _{n \rightarrow \infty} f_{n}(\beta)=F_{s}$. Then $F_{s} \in L$ since it is in the derived set of an orbit.

If $i\left(\alpha_{1}, \alpha_{2}\right) \neq 0$, fill out $\alpha_{1}$ to an admissible system $\bar{\alpha}$. By lemma $1.1 \alpha_{2}$ satisfies (ii) for some $\alpha_{j} \in \bar{\alpha}$. As in that lemma replace $\alpha_{j}$ with $\alpha_{j}^{\prime}$ so that $i\left(\alpha_{2}, \bar{\alpha}^{\prime}\right)<i\left(\alpha_{2}, \bar{\alpha}\right)$. But $\alpha_{j}^{\prime}$ can be joined by a path in $L$ to any curve in $\bar{\alpha}$. The induction step is complete.

Next fix an admissible system $\bar{\alpha}$ and find a curve $\beta$ with the property that if $\sigma$ is any segment that leaves an $\alpha_{j}$ and then returns to that $\alpha_{j}$ in the opposite direction, $\sigma \cap \beta \neq \varnothing$. That such a $\beta$ exists one can see as follows. Complete $\bar{\alpha}$ to a system of $3 g-3$ disjoint curves in $S_{t}$ that divide $M$ into $2 g-2$ 'pairs of pants'. Find a foliation $F$ such that for any three curves that bound a pair of pants, the transverse length of one is always less than the sum of the other two. This is the triangle inequality situation in [1]. 


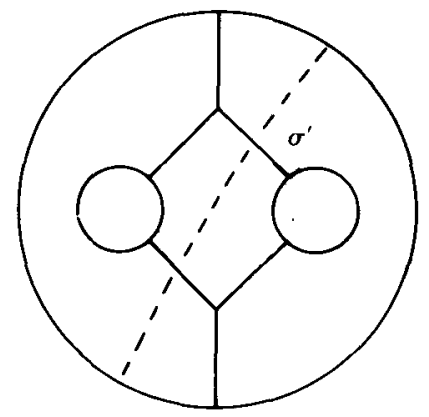

FIGURE 3

Since $S$ is dense in $\mathscr{P} \mathscr{F}$ we can certainly find a curve $\beta$ with the same property. Now if $\sigma^{\prime}$ is any segment leaving one of these boundary components and returning as shown, then $\sigma^{\prime} \cap \beta \neq \varnothing$. Any $\sigma$ must contain such a $\sigma^{\prime}$ as a subset. Any $\beta_{n}$ sufficiently close to $\beta$ has the same property and thus by lemma 1.1 no sufficiently close $\beta_{n}$ can be in $L$. For if $\beta_{n}$ left and returned to $\alpha_{j}$ as in that lemma, it would be disjoint from a segment that did the same. Thus $\beta \notin L, L \neq \mathscr{P} \mathscr{F}$ and we have some open set $U$ disjoint from $L$. We can assume $i\left(F, \alpha_{1}\right) \neq 0$ for all $F \in U$. If $L$ contains any open $U^{\prime}$, find a non-dividing curve $\alpha \in S_{\mathrm{t}} \cap U^{\prime}$ and $f \in \operatorname{Mod}\left(H_{g}\right)$ such that $f(\alpha)=\alpha_{1}$. Then $f\left(U^{\prime}\right)$ is a neighbourhood of $\alpha_{1}$ in $\mathscr{P} \mathscr{F}$. But $\tau_{\alpha_{1}}^{n}\left(U_{1}\right) \subset f\left(U^{\prime}\right)$ for large $n$. Thus $f^{-1} \tau_{\alpha_{1}}^{n}(U) \subset U^{\prime}$. This contradicts the invariance of $L$.

2. Theorem 1.2 and its proof suggest which foliations should be in the domain of discontinuity. This section makes that precise. We first recall some definitions.

A foliation is arational if no simple curve is represented by critical leaves. This implies all leaves not hitting a critical point are dense. A foliation $F$ is uniquely ergodic if it is arational and any topologically conjugate foliation is measure equivalent to a multiple of $F$.

THEOREM B [6]. Almost all foliations in $\mathscr{P} \mathscr{F}$ are uniquely ergodic.

'Almost all' refers to the canonically defined measure class $m$ in $\mathscr{P} \mathscr{F}$. Let $T=$ $\{G \mid i(F, G)=0$ some $F \in L\}-L$.

Corollary. $m(T)=0$.

The following example for $g=2$ shows $T=\varnothing$. The foliation $G$ has $\beta$ as a closed singular leaf. By definition $i(G, \beta)=0$ and $\beta \in L$. We show $G \notin L$ by showing that for any simple $\gamma$ near $G, \gamma \notin S_{\text {. }}$.

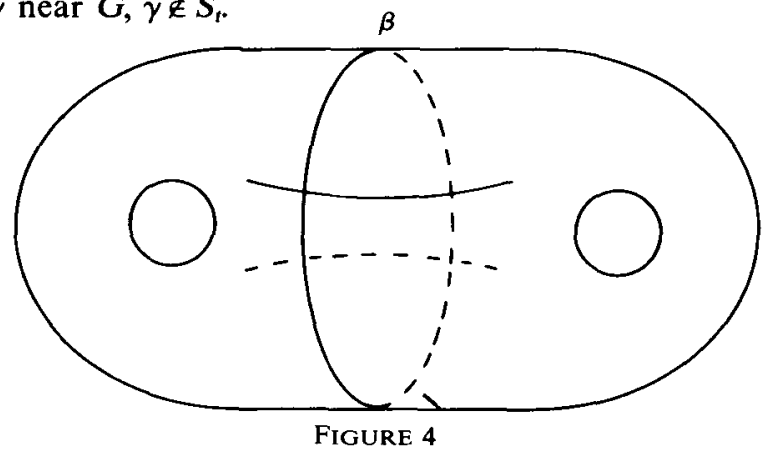


Let $\alpha_{1}, \alpha_{2}, \alpha_{3} \in S_{t}$ satisfy $i\left(\beta, \alpha_{1}\right)=2, i\left(\beta, \alpha_{i}\right)=0, i=2,3$ and $\alpha_{i}$ bound two pairs of pants. The foliation $G$ in terms of the pants is given by figure 5 .
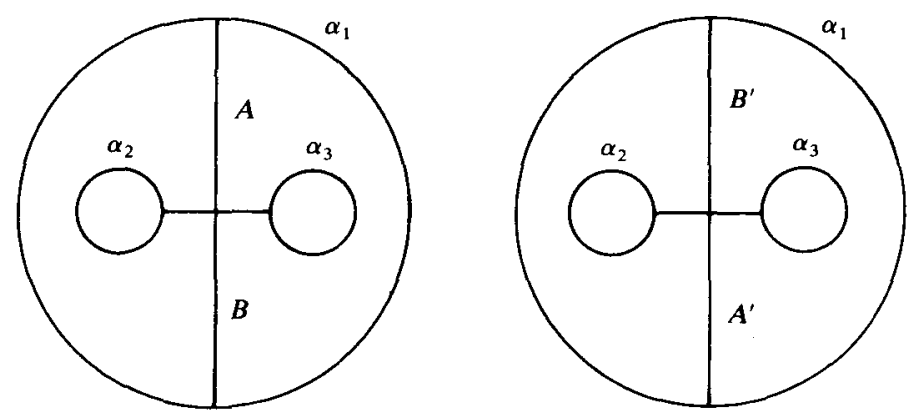

FIGURE 5

The leaf $A$ is joined to $A^{\prime}$ and $B$ is joined to $B^{\prime}$ across $\alpha_{1}$ to form $\beta$. Now if $\gamma \in S_{\imath}, \gamma$ is represented by a foliation as in figure 6 . The width of the strips are equal in the two pairs of pants, and for $\gamma$ near $G$, the widths are small.
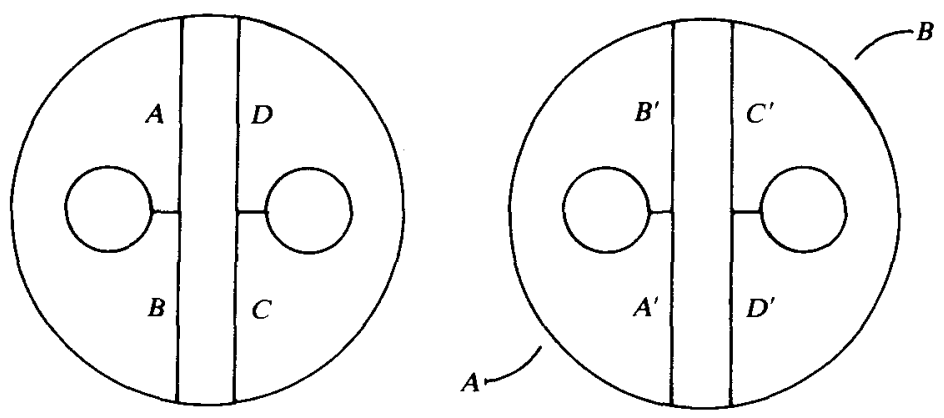

Figure 6

Now $A$ and $D$ must cross $\alpha_{1}$ near $A^{\prime}$ and $D^{\prime}$. If for instance $A$ next crosses $\alpha_{2}$ after crossing $\alpha_{1}$, as in figure 6 , then $B$ either next crosses $\alpha_{1}$ again or next crosses $\alpha_{3}$ after crossing $\alpha_{1}$ as in figure 6. It is easy to check in either case that if $\alpha_{1}$ is removed, there is no leaf for either $\alpha_{2}$ or $\alpha_{3}$ satisfying (ii) of lemma 1.1. Thus $\gamma$ can't be in $S_{t}$. The case of $D$ next crossing $\alpha_{3}$ after $\alpha_{1}$ is similar.

Now fix $\bar{\alpha}$ admissible and consider foliations on $X-\left\{\alpha_{1}, \ldots, \alpha_{g}\right\}$. We assume $i\left(F, \alpha_{i}\right) \neq 0$ all $i$ so $\alpha_{i}$ is taken to be transverse to $F$. Let $A_{\bar{\alpha}}=\left\{F \mid i\left(F, \alpha_{i}\right) \neq 0\right.$ all $i$ and there is no leaf crossing $\alpha_{j}$ in one direction and then $\alpha_{j}$ in the oppsoite direction. $\}$ An example is given in figure 7. 


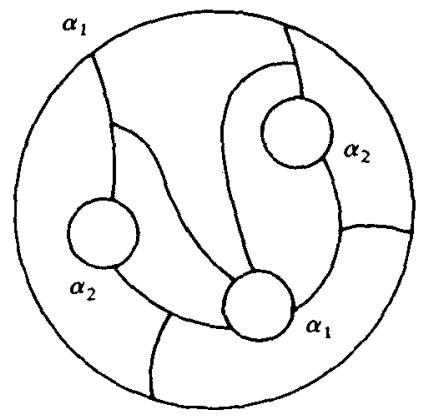

FIGURE 7

This is the generalization to a $2 g$-holed sphere of the triangle inequality condition on a pair of pants.

A system $|\alpha|=\left(\alpha_{1}, \ldots, \alpha_{6 g-6}\right)$ of curves in $S_{t}$ is complete if

(i) no $\alpha_{i}$ is dividing;

(ii) $\alpha_{1}, \ldots, \alpha_{3 g-3}$ divide $M$ into pants;

(iii) $i\left(\alpha_{j}, \alpha_{j-3 g-3}\right)=2 ; i\left(\alpha_{j}, \alpha_{k}\right)=0$ for $j \geq 3 g-2, k \leq 3 g-3, k \neq j-3 g-3$.

For any $F$ and $|\alpha|$ let

and

$$
i(F,|\alpha|)=\sum_{i=1}^{6 g-6} i\left(F, \alpha_{i}\right)
$$

$$
D_{|\alpha|}=\{F \mid i(F,|\alpha|) \leq i(F,|\beta|) \quad \text { all complete }|\beta|\}
$$

Let

$$
\mathcal{O}=\left\{F: F \in A_{\bar{\alpha}} \text { some } \bar{\alpha} \text { and } i(F, G) \neq 0 \text { for all } G \in L\right\} .
$$

Notice $O \cap(T \cup L)=\varnothing$. Part of $\S 3$ is devoted to showing $O \cup T \cup L=\mathscr{P} \mathscr{F}$.

THEOREM 2.1. Mod $\left(H_{g}\right)$ and $\operatorname{Mod}_{0}\left(H_{g}\right)$ act properly discontinuously on $O$ which is a non-empty open set. In fact $D_{\alpha \mid} \cap O$ is a fundamental domain for the action of $\operatorname{Mod}\left(H_{g}\right)$ on $O$ in the sense that any $F \in O$ is $\operatorname{Mod}\left(H_{g}\right)$ equivalent to a point in $D_{\alpha \mid}$ and any $F \in D_{\alpha \mid} \cap O$ is equivalent to only finitely many others.

Proof. Since $L$ is closed and $i(\cdot, \cdot)$ is continuous, $T \cup L$ is also closed. Since $L$ has empty interior and $T$ has measure zero, $T \cup L$ has empty interior. Each $A_{\bar{\alpha}}$ is open and non-empty so $A_{\bar{\alpha}}-(T \cup L)$ is also. Thus $O$ is non-empty. Let $F \in O$ and suppose $F \notin D_{\alpha \mid}$ for any $|\alpha|$. Then there is an infinite sequence of $\left|\alpha_{n}\right|$ with $i\left(F,\left|\alpha_{n}\right|\right)$ decreasing. For some sequence $\alpha_{n}$ of curves belonging to $\left|\alpha_{n}\right|, \alpha_{n}$ is unbounded in $\mathscr{M F}$. Since $\mathscr{P} \mathscr{F}$ is compact this means there exists a subsequence again denoted $\alpha_{n}$ and a sequence $r_{n} \in \mathbb{R}^{+}$with $\lim _{n \rightarrow \infty} r_{n}=0$ such that

$$
\lim _{n \rightarrow \infty} r_{n} \alpha_{n}=G, \quad G \in L \text {. }
$$

But now $i\left(F, \alpha_{n}\right)$ bounded so $i(F, G)=0$ and we have a contradiction. Essentially the same argument shows that a compact set $K \subset \mathcal{O}$ intersects only finitely many $D_{\alpha \uparrow}$.

Now suppose $f_{n}(K) \cap K \neq \varnothing$ for infinitely many $f_{n} \in \operatorname{Mod}\left(H_{g}\right)$ where $K \subset \mathcal{O}$ compact in $\mathscr{P} \mathscr{F}$. Choosing subsequences we have $F_{n} \in K$ converging in $\mathscr{P} \mathscr{F}$ to $F_{0} \in K$ 
such that $f_{n}\left(F_{n}\right) \in K$ converges to $G_{0}$. Again choosing further subsequences we can assume $F_{n} \in D_{\alpha \mid}$ and $f_{n}\left(F_{n}\right) \in D_{|\beta|}$. Then

$$
\begin{aligned}
i\left(f_{n}\left(F_{n}\right),|\beta|\right) & \leq i\left(f_{n}\left(F_{n}\right), f_{n}(|\alpha|)\right)=i\left(F_{n},|\alpha|\right) \\
& \leq i\left(F_{n}, f_{n}^{-1}(|\beta|)\right)=i\left(f_{n}\left(F_{n}\right),|\beta|\right) .
\end{aligned}
$$

Since $F_{n} \in K$, this implies $|\alpha|=f_{n}^{-1}|\beta|$ for infinitely many $n$. But there are only finitely many $f$ mapping one complete system to another. This shows $\operatorname{Mod}\left(H_{g}\right)$, hence also $\operatorname{Mod}_{0}\left(H_{g}\right)$, act discontinuously on $\mathcal{O}$.

Suppose $F \in \mathscr{O} \cap D_{|\beta|}$. There exists $f \in \operatorname{Mod}\left(H_{g}\right)$ such that $f(|\beta|)=|\alpha|$. Then $f(F) \in$ $D_{|\alpha|}$. Finally if $F \in D_{\alpha \mid}$ and $f_{n}(F) \in D_{|\alpha|}$ for infinitely many $n$,

$$
\begin{aligned}
i\left(f_{n}(F),|\alpha|\right) & \leq i\left(f_{n}(F), f_{n}(|\alpha|)\right)=i(F,|\alpha|) \\
& \leq i\left(F, f_{n}^{-1}(|\alpha|)\right)=i\left(f_{n}(F),|\alpha|\right) .
\end{aligned}
$$

This implies $i(F,|\alpha|)=i\left(F, f_{n}^{-1}(|\alpha|)\right)$ and since $i(F, G) \neq 0$ for all $G \in L, f_{n}^{-1}(|\alpha|)$ cannot represent an infinite sequence as before. This finishes the proof.

The next lemma shows that at least at some points of $T, \operatorname{Mod}_{0}\left(H_{g}\right)$ does not act discontinuously.

LeMMA 2.2. If $i(F, \alpha)=0$ for some $\alpha \in S_{t}$, then $\operatorname{Mod}_{0}\left(H_{g}\right)$ hence $\operatorname{Mod}(g)$ do not act discontinuously at $F \in \mathscr{P} \mathscr{F}$.

Proof. Let $\tau_{\alpha}$ be the Dehn twist about $\alpha$. If $i(\beta, \alpha)=0$ then $\tau_{\alpha}^{-1}(\beta)=\beta$ so

$$
i\left(\tau_{\alpha}(F), \beta\right)=i\left(F, \tau_{\alpha}^{-1}(\beta)\right)=i(F, \beta) .
$$

If $i(\beta, \alpha) \neq 0, i(F, \beta)$ is realized by a curve transverse to $\alpha, \tau_{\alpha}^{-1}(\beta)$ is realized by the same transverse arcs together with arcs of $\alpha$ which have zero transverse length so

$$
i\left(\tau_{\alpha}(F), \beta\right)=i\left(F, \tau_{\alpha}^{-1}(\beta)\right)=i(F, \beta) .
$$

Thus $\tau_{\alpha}$ fixes $F$ and has infinite order and the lemma follows.

Remark. The example of figure 4 thus shows that $L \neq$ closure of the set of foliations fixed by infinite cyclic subgroups of $\operatorname{Mod}\left(H_{g}\right)$ since that foliation $G$ is fixed by the Dehn twist about $\beta$.

3. We next analyze the limit set $L$. The key is the reduction step in lemma 1.1. We will apply this to a generic foliation in $L$.

Suppose again the system $\bar{\alpha}=\left(\alpha_{1}, \ldots, \alpha_{g}\right)$ is admissible and consider $X=$ $M-\left\{\alpha_{1}, \ldots, \alpha_{g}\right\}$. Let $\rho$ be a segment which leaves a side say, $\alpha_{1}^{+}$, of $X$ and crosses to $\alpha_{1}^{+}$again. Let $B_{\bar{\alpha}, \rho}=\{F \mid$ when restricted to $X, F$ has a leaf in the homotopy class of $\rho$ \}. Here homotopy is relative to the boundary. The leaf may consist of segments from $\alpha_{1}^{+}$to singularities together with saddle connections. Such leaves we call returning.

It is clear that modulo the action of $\operatorname{Diff}_{+}(X) / \operatorname{Diff}_{0}(X)$ there are only finitely many homotopy classes of $\rho$ (for fixed $\bar{\alpha}$ ) and thus finitely many $B_{\bar{\alpha}, \rho}$. These are called basic sets. Figure 8 illustrates an example in genus 2. 


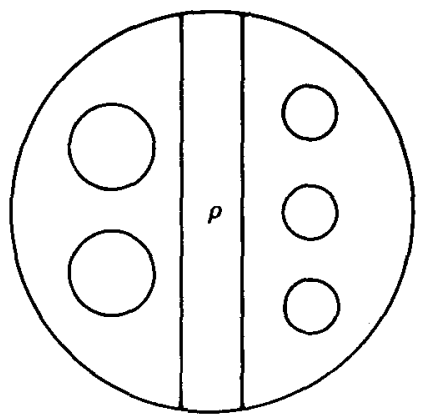

Figure 8

LEMMA 3.1. Fix $\bar{\alpha}$ and let $B_{\bar{\alpha}}=\bigcup_{\rho} B_{\bar{\alpha}, \rho}$ the union over all foliations with some returning leaf. Then $B_{\bar{\alpha}} \cup\left\{F \mid i\left(F, \alpha_{i}\right)=0\right.$ some $\left.\alpha_{i}\right\}$ is closed.

Proof. Its complement is the set $\boldsymbol{A}_{\bar{\alpha}}$ defined in the last section which is clearly open.

Define $L^{\prime} \subset \mathcal{M} \mathscr{F}$ to be the set of foliations $F$ such that either

(i) $i(F, \alpha)=0$ for some $\alpha \in S_{i}$; or

(ii) if $i(F, \alpha) \neq 0$ for all $\alpha \in S_{t}$, then for any admissible $\bar{\alpha}, F \in B_{\tilde{\alpha}}$.

Note again $L^{\prime}$ is well-defined as a subset of $\mathscr{P} \mathscr{F}$.

\section{Proposition 3.2. $L \subset L^{\prime}$}

Proof. The proof is essentially that of lemma 1.1. If $\gamma \in S_{t}$ then $\gamma \in L^{\prime}$ since $i(\gamma, \gamma)=0$. Next suppose $F_{0}=\lim _{n \rightarrow \infty} \gamma_{n}, \gamma_{n} \in S_{t}$, convergence in $\mathscr{P} \mathscr{F}$. Suppose $i\left(F_{0}, \alpha\right) \neq 0$ for all $\alpha$ and $\bar{\alpha}=\left(\alpha_{1}, \ldots \alpha_{g}\right)$ admissible. We have $i\left(\gamma_{m}, \alpha_{i}\right) \neq 0$ for large $n$. By lemma 1.1, $\gamma_{n} \in B_{\bar{\alpha}}$. By lemma 3.1 either $F_{0} \in B_{\bar{\alpha}}$ or $i\left(F, \alpha_{i}\right)=0$ for some $i$. By hypothesis the first possibility must occur, and the proposition follows.

Now suppose $F_{0} \in L^{\prime}$ satisfies (ii) of the definition. Say $\alpha_{1}^{+}$has a returning leaf. We look for the smallest subset of the set of homotopy classes of returning leaves for $\alpha_{1}^{+}$with the property that any segment in $X$ from $\alpha_{1}^{+}$to $\alpha_{1}^{-}$that does not intersect a homotopy class in this subset also does not intersect any of the other returning leaves from $\alpha_{1}^{+}$. Figure 9 depicts two possibilities with the elements of the subset denoted $*$.
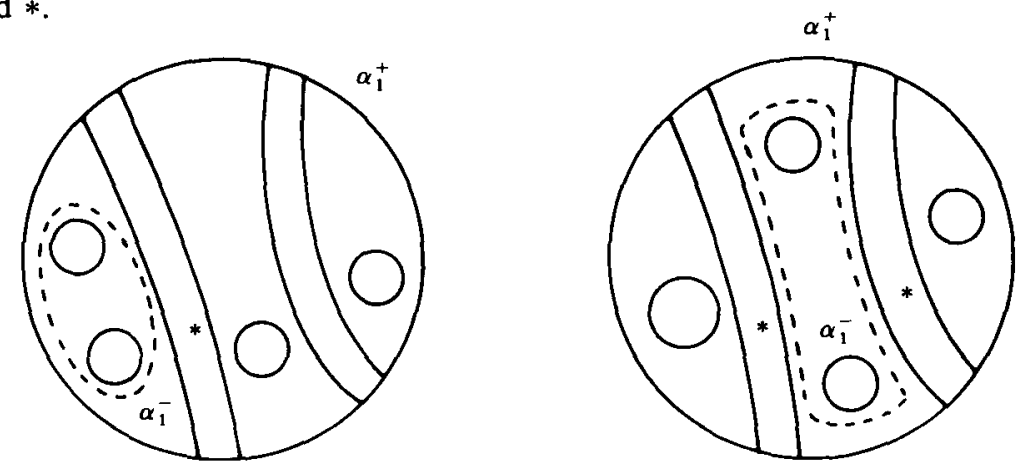

Figure 9 
Let $\alpha_{1}^{*}$ be the closed curve homotopic to the union of these distinguished leaves and one or two segments of $\alpha_{1}$ as indicated in figure 9 . In either case we have a lemma whose proof is identical to the one given in $\S 1$.

Lemma 3.2. $i\left(F_{0}, \alpha_{1}^{*}\right)<i\left(F_{0}, \alpha_{1}\right)$. Replace $\alpha_{1}$ with $\alpha_{1}^{*}$. The new system of curves $\bar{\alpha}^{*}$ is admissible.

Now since $F_{0} \in L^{\prime}, F_{0} \in B_{\bar{\alpha}^{*}, \rho^{*}}$ for a non-empty finite set of $\rho^{*}$. Choose one returning class and repeat the procedure to find a sequence $\bar{\alpha}^{(n)}$ and for each $n$, a finite set

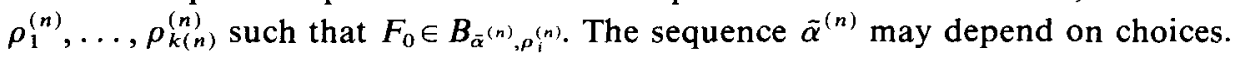

Proposition 3.3. Suppose $F_{0} \in L^{\prime}$ satisfies (ii) of the definition. Then for each sequence $\rho_{i}^{(n)}$,

$$
\bigcap_{n=1}^{\infty} B_{\bar{\alpha}^{(n)}, \rho_{i}^{(n)}}=\left\{F: i\left(F, F_{0}\right)=0\right\}
$$

and the intersection of this set with $L$ is non-empty. If $F_{0}$ is uniquely ergodic, then this intersection equals $F_{0}$ and $F_{0} \in L$.

Proof. It is obvious the sequence $\bar{\alpha}^{(n)}$ is not finite; there is a subsequence of $\alpha_{i}^{(n)} \in \bar{\alpha}^{(n)}$ for some $i$ and a sequence $r_{n} \in \mathbb{R}$ going to zero with

$$
\lim _{n \rightarrow \infty} r_{n} \alpha_{i}^{(n)}=G \in L
$$

But $\lim _{n \rightarrow \infty} r_{n} i\left(F_{0}, \alpha_{i}^{(n)}\right)=0$ since $i\left(F_{0}, \alpha_{i}^{(n)}\right)$ is non-increasing. By the continuity of $i(\cdot, \cdot), i\left(F_{0}, G\right)=0$. Now infinitely often some $\rho_{i}^{(n+1)}$ is a proper subset of a $\rho_{j}^{(n)}$ which is a returning leaf for a curve being replaced. This means there is a sequence $\rho_{i}^{(n)}$ whose length goes to infinity with $n$. These leaves must eventually intersect every curve and this implies that any $\alpha$ which occurs in an admissible system is eventually replaced. Thus all sequences $\rho_{j}^{(n)}$ have lengths going to infinity.

Now for a sequence $\rho_{i}^{(n)}$ and any homotopy class $\beta \in S$, consider the minimal number of intersections of transverse $\beta \in S$ with $\rho_{i}^{(n)}$. This must go to infinity with $n$ since the length of $\rho_{i}^{(n)}$ goes to infinity and $F_{0}$ is arational. This minimal number

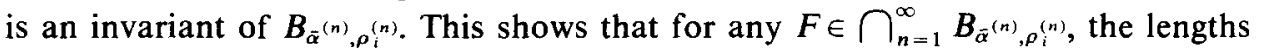
of $\rho_{i}^{(n)}$ go to infinity and $F$ arational. If $i\left(F, \alpha_{i}^{(n)}\right)$ were bounded below away from zero we would have a sequence of leaves, leaving segments with transverse length bounded away from zero travelling distances going to infinity before returning to the segments. This is impossible in an arational foliation. Thus $\lim _{n \rightarrow \infty} i\left(F, \alpha_{i}^{(n)}\right)=0$ which implies $i(F, G)=0$ and hence $i\left(F, F_{0}\right)=0$. On the other hand, if $i\left(F, F_{0}\right)=0, F$ is topologically equivalent to $F_{0}$. Thus $F \in B_{\bar{\alpha}^{(n)}, \rho_{i}^{(n)}}$ for each such set.

Corollary 3.4. $L^{\prime} \subset L \cup T, m\left(L^{\prime}-L\right)=0, \mathcal{O} \cup L \cup T=\mathscr{P} \mathscr{F}$.

Proof. If $F_{0} \in L^{\prime}$ satisfies (i) of the definition, then $F_{0} \in L \cup T$. If $F_{0}$ satisfies (ii), then $i\left(F, F_{0}\right)=0$ for some $F \in L$ by proposition 3.3 so again $F_{0} \in L \cup T$. The second statement of the corollary follows from the fact that $m(T)=0$. Finally, if $F_{0} \notin \mathcal{O}$, then either $i\left(F, F_{0}\right)=0$ for some $F \in L$ or $F_{0} \in B_{\tilde{\alpha}}$ for each admissible $\bar{\alpha}$. Therefore, $F_{0} \in L \cup T \cup L^{\prime}=L \cup T$. 
Corollary 3.5. Each $B_{\bar{\alpha}, \rho}$ contains an open set $\hat{O}_{\bar{\alpha}, \rho} \subset \mathcal{O}$.

Proof. $L \cup T$ has empty interior and $\mathscr{P} \mathscr{F}=\mathscr{O} \cup L \cup T$.

We summarize our results in this section as:

THEOREM 3.6. For given $\bar{\alpha}$ admissible there exist sets $B_{\bar{\alpha}, p_{i}}$ and $\mathcal{O}_{\bar{\alpha}, p_{i}} \subset \mathcal{O} \cap B_{\bar{\alpha}, \rho_{i}}$, $i=1, \ldots, k$ such that given $\varepsilon>0$, there is a cover of the uniquely ergodic part of $L$ which is almost all of $L$ by $\operatorname{Mod}\left(H_{g}\right)$ images of $B_{\bar{\alpha}, \rho_{i}}$ of diameter less than $\varepsilon$. Each such image contains the image of $\mathcal{O}_{\bar{\alpha}, \rho_{i}}$ which is in $\mathcal{O}$.

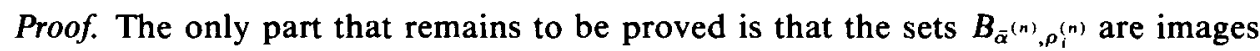
of a finite set of $B_{\bar{\alpha}, \rho}$. But this follows from the fact that there is $f \in \operatorname{Mod}\left(H_{g}\right)$ such that $f(\bar{\alpha})=\bar{\alpha}^{(n)}$. Then by composing $f^{-1}$ with twists about $\alpha_{i}$ one can place $f^{-1}\left(\rho_{i}^{(n)}\right)$ in one of a finite set of returning classes for $\bar{\alpha}$.

4. For the rest of the paper we restrict to $g=2$. Label the curves of $\bar{\alpha}$ as $\alpha_{1}, \alpha_{2}$ and suppose there is a returning leaf $\rho$ from $\alpha_{1}^{+}$to itself as in figure 10.

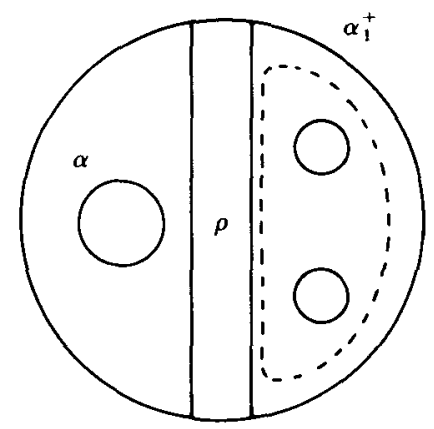

Figure 10

Consider the dotted curve $\alpha_{1}^{*}$ uniquely determined by $\rho$. Then $\alpha_{1}, \alpha_{1}^{*}$, and $\alpha$ bound a pair of pants and

$$
i\left(F, \alpha_{1}\right)>i(F, \alpha)+i\left(F, \alpha_{1}^{*}\right) .
$$

This implies $\alpha=\alpha_{2}$. The other pair of pants bounded by $\alpha_{1}, \alpha_{1}^{*}$, and $\alpha_{2}$ must satisfy the same inequality so there must be a returning leaf from $\alpha_{1}^{-}$to itself as well.

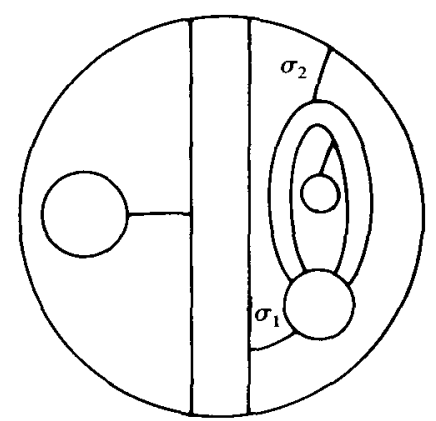

Figure 11 
The segments $\sigma_{1}$ and $\sigma_{2}$ which join $\alpha_{1}^{+}$and $\alpha_{1}^{-}$respectively to singularities are defined up to a Dehn twist about $\alpha_{1}^{*}$.

For given $\bar{\alpha}$ the sets $B_{\bar{\alpha}, \rho}$ are disjoint for different $\rho$. This is not true if $g \geq 3$ and is the reason the picture developed in this section does not work for $g \geq 3$.

Replace $\alpha_{1}$ with $\alpha_{1}^{*}$ to form $\bar{\alpha}^{*}=\left(\alpha_{1}^{*}, \alpha_{2}\right)$. Then $B_{\bar{\alpha}, \rho}$ is a union of sets $B_{\bar{\alpha}^{*}, \rho^{*}}$ for different $\rho^{*}, A_{\bar{\alpha}^{*}}$, and a subset of the set of positive codimension defined by $i\left(F, \alpha_{2}\right)=0 ; B_{\bar{\alpha}^{*}, \rho^{*}}$ and $A_{\bar{\alpha}^{*}}$ are disjoint and $A_{\bar{\alpha}^{*}} \subset \mathcal{O} ; B_{\bar{\alpha}^{*}, \rho^{*}}$ is the $\operatorname{Mod}\left(H_{2}\right)$ image of $B_{\bar{\alpha}, \rho}$ and $A_{\bar{\alpha}^{*}}$ is the image of $A_{\bar{\alpha}}$. Thus together with theorem 3.6 we have:

THEOREM 4.1. For $g=2$, the limit set $L$ is up to measure zero the result of removing successively disjoint $\operatorname{Mod}\left(\mathrm{H}_{2}\right)$ images of a given $\boldsymbol{A}_{\bar{\alpha}}$ from $\mathscr{P} \mathscr{F}$.

5. The description of $L$ in $\S 4$ allows us to prove:

THEOREM 5.1. $m(L)=0$ for $g=2$.

At each stage of the construction of $L$ we have sets $B$ and $A \subset B \cap O$. We would like to show $m(A) / m(B) \geq c>0$ for some $c>0$ independent of the stage. This however appears not to be true. However it is enough to show such an inequality is true infinitely often for almost all $F$.

Proposition 5.2. Suppose there exists a set $E \subset \mathscr{P} \mathscr{F}$ and $c>0$ with the property that given $\varepsilon>0$ there is a disjoint cover of $E$ except for a set of measure 0 by sets $V_{i}$ of diameter $<\varepsilon$ such that each $V_{i}$ contains a set $U_{i}$ disjoint from $E$ and $m\left(U_{i}\right) / m\left(V_{i}\right) \geq c$. Then $m(E)=0$.

Proof. Suppose $m(E)=m_{0}>0$. Take the disjoint cover small enough so its union $V$ satisfies

$$
\left(\frac{1}{c}-1\right) m(V-E) \leq m_{0} / 2
$$

Let $U$ be the union of the corresponding $U_{i}$. Then $m(U) / m(V) \geq c$. However $U \subset V-E$. Thus

$$
\begin{aligned}
m_{0}=m(E) & \leq m(V)-m(U) \leq m(U)\left(\frac{1}{c}-1\right) \\
& \leq m(V-E)\left(\frac{1}{c}-1\right) \leq m_{0} / 2
\end{aligned}
$$

and we have a contradiction.

Denote by $\Sigma$ the standard simplex in $\mathbb{R}^{n}$ defined by $\sum_{i=1}^{n} \lambda_{i}=1, \lambda_{i} \geq 0$. If $A: \mathbb{R}^{n} \rightarrow \mathbb{R}^{n}$ is a matrix with non-negative entries denote by $\bar{A}: \Sigma \rightarrow \Sigma$ the corresponding projective linear map. The Jacobian of $\bar{A}$ at $x$ is $|A x|^{-n}$. Following [4] we say $A$ is $C$ distributed if the columns $A_{i}$ of $A$ satisfy $\left|A_{i}\right| /\left|A_{j}\right| \leq C$. If the columns of $A$ are $C$ distributed it is easy to see that the ratio of the Jacobian of $\bar{A}$ at any two points is bounded by $C^{n}$.

We will find the desired cover of $L$ in the context of train tracks. As we will see each set in the cover will be a subset of a set of the form $B_{\bar{\alpha}, \rho}$. 
At this point we recall the basic concept of a train track. Our exposition is taken from [3] and [4]. The original ideas appear in [9]. A train track $\tau$ on a surface $X$ is a connected one dimensional cell complex made up of vertices called switches and edges called branches disjointly embedded in $X$. We require that

(1) at each switch $\tau$ is trivalent;

(2) $\tau$ is $C^{1}$ away from the switches;

(3) for each switch $v$ the tangent lines to $\tau$ at $v$ using one-sided limits agree along branches incident to $v$. One branch at the switch has the property that a curve on $\tau$ through $v$ and that branch is $C^{1}$ at the switch. We call it the larger branch at $v$.

(4) Each component of the complement of $\tau$ is a 3-gon, an open 2-disc with 3 discontinuities in the tangent lines to $F r D$.

Train tracks satisfying (1)-(4) are called complete. We say $\tau$ carries a foliation $F$ if there is a map $\phi:(X$-singularities $F)$ onto $\tau$ homotopic to the identity such that $d \phi_{p} \mid$ (tangent line to $F$ ) is non-zero for every $p$. A complete track carries an open set of foliations in $M \mathscr{F}$.

We will consider two train tracks to be equivalent if one can get to the other by a series of shifts as in figure 12 .



Figure 12

Shift equivalent tracks carry the same foliations.

A track with weights assigns to each branch a positive number such that at the vertices the switch condition is satisfied: the weight of the larger branch equals the sum of the other two.

A train track $\tau$ with weights satisfying the switch condition uniquely determines a measured foliation carried by $\tau$. One thinks of running leaves along the branches with transverse measure given by the weights. The set of weights, projectivized, satisfying the switch conditions defines a polyhedral surface in the simplex $\Sigma$. It is also clear that there are only finitely many combinatorial types of tracks, defining polyhedra $H_{1}, \ldots, H_{k}$.

The basic operation on weighted train tracks is the splitting operation. A branch which is the larger edge at each switch we call a winged branch, see figure 13. If $a>c$ in figure 13 ( $d>b$ because of the switch condition) the operation is to split the winged branch as follows.

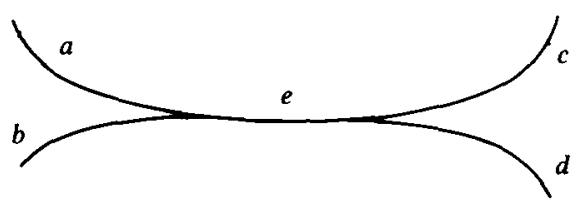

FIGURE 13

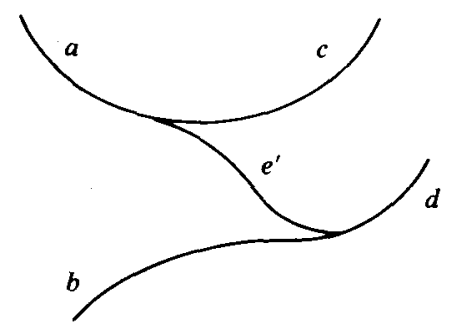


If $a<c(b>d)$ the splitting is:
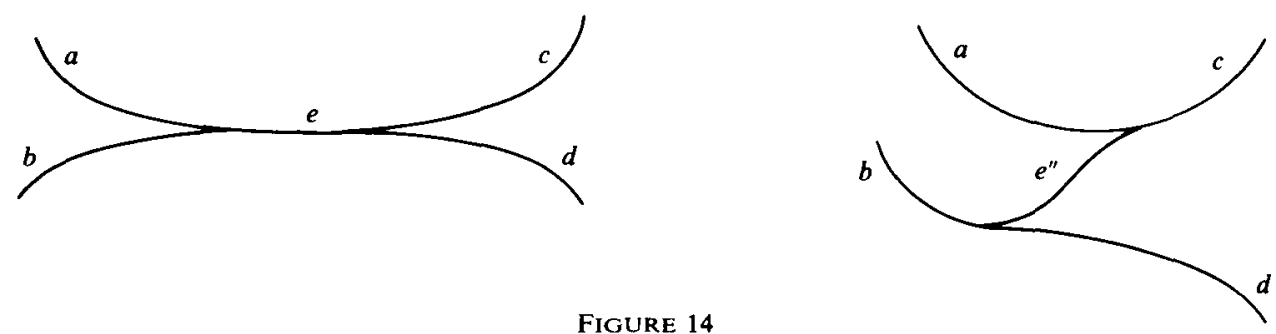

FIGURE 14

The weights of $\tau^{\prime}, \tau^{\prime \prime}$ are related to the weights of $\tau$ by

$$
\begin{aligned}
& e=e^{\prime}+a+d, \\
& e=e^{\prime \prime}+b+c,
\end{aligned}
$$

resp. As indicated in [4] one thinks of $\tau^{\prime}, \tau^{\prime \prime}$ as embedded in a regular neighbourhood of $\tau$. The formulae are realized geometrically by mapping the new tracks onto $\tau$. Every foliation carried by $\tau^{\prime}$ or $\tau^{\prime \prime}$ is carried by $\tau$ and every foliation carried by $\tau$ is carried by either $\tau^{\prime}$ or $\tau^{\prime \prime}$. (We ignore the possibility of $a=c$ as these foliations form a set of measure zero.) The choice of which way to alter the track is determined by the topological condition of whether the foliation has a leaf going from $a$ to $d$ or $b$ to $c$. The formulae give (projective) linear maps from a pair $H_{i}, H_{j}$ into $\Sigma$ and the two images divide the original subset $H_{k}$ satisfying the switch conditions on $\tau$ into two pieces.

For any minimal $F$ carried by $\tau$ we repeat this procedure and get what Kerckhoff calls a simplicial system for $F$. This consists of a finite number of states, the combinatorial types of tracks together with a numbering of the branches, and an infinite sequence of symbols where each symbol is a projective linear map of $\Sigma$ which is a product (*) of two elementary matrices. Let $A_{n}$ be the product of the first $n$ symbols. The sequence of $A_{n}$ is called the expansion of $F$. The idea of associating a matrix expansion to the closely related interval exchange is due to Veech [10].

The special nature of foliations in $L$ indicates that we should be interested only in certain kinds of tracks $\tau$. We require that there be an admissible system $\bar{\alpha}=\left(\alpha_{1}, \alpha_{2}\right)$ such that each $\alpha_{i}$ is embedded in $\tau$ and has exactly two switches on it with one branch leaving from each side. We do not require the embedding to be $C^{1}$ but we do require that if it is not $C^{1}$ embedded, it have two corners. This means any leaf running along a branch to $\alpha_{i}$ will actually cross $\alpha_{i}$.

We say $\tau$ is admissible for $\bar{\alpha}$. There are three types of tracks admissible for a given $\bar{\alpha}$.

(I) Every foliation carried by $\tau$ has a return leaf to either $\alpha_{1}$ or $\alpha_{2}$.

(II) No foliation carried by $\tau$ has a return leaf to either $\alpha_{1}$ or $\alpha_{2}$.

(III) $\tau$ carries a foliation with a returning leaf and a foliation with no return leaf. The foliations carried by tracks of type (I) are in $B_{\bar{\alpha}, \rho}$ for some $\rho$ and those carried by tracks of type (II) are in $\boldsymbol{A}_{\bar{\alpha}}$. 
We will assign a simplicial system to every minimal $F \in L$ and will need two lemmas to show that only these tracks (I)-(III) need by considered.

LemmA 5.3. Suppose $\tau$ is type (III) with respect to $\bar{\alpha}$. Then there exists a winged branch $\sigma$ joining two distinct switches neither of which is on either $\alpha_{i}$.

Proof. Since $\tau$ is type (III) it carries a foliation with returning leaves to both sides of $\alpha_{1}$ say. Pick one side. There is therefore a $C^{1}$ arc $\beta$ in $\tau$ beginning and ending at the same switch on $\alpha_{1}$ not otherwise intersecting either $\alpha_{i}$. Since there is only one branch leaving each side of $\alpha_{1}$, by assumption, $\beta$ must travel along a maximal connected set of branches $\rho$ leaving $\alpha_{1}$, then travel around a loop $\gamma$ and then return along $\rho$.

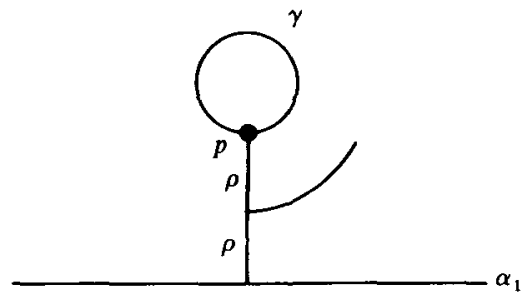

FIGURE 15

Call $p$ the switch on $\rho$ incident to $\gamma$. The branch on $\rho$ must be larger at $p$ so that $\beta$ forms a $C^{1}$ curve from $\rho$ to $\gamma$ and back to $\rho$. If there is a branch on $\rho$ between $\alpha_{1}$ and $p$ which is larger at a switch closer to $\alpha_{1}$ but not on $\alpha_{1}$, we are done, for then there must be a winged branch on $\rho$. Suppose there is no such branch. Then every leaf from any point on $\rho$ travelling toward $\alpha_{1}$ must cross $\alpha_{1}$.

Orient the loop $\gamma$ with a + and - direction. Travelling in the + direction along $\gamma$, consider the set of branches $\tilde{\gamma}$ on $\gamma$ with the property that any $C^{1}$ curve through a point of $\tilde{\gamma}$ in the + direction must cross $\alpha_{1}$. The last branch of $\gamma$ in the + direction has this property so the set is non-empty. Let $\gamma_{+}$be the first branch in the + direction in this set. Similarly find $\gamma_{-}$.

Travelling in the + direction, $\gamma_{+}$cannot come before $\gamma_{-}$for at the switch at the end of $\gamma_{+}$, the branch on $\gamma$ following $\gamma_{+}$must be larger. This contradicts the definition of $\gamma_{-}$. If $\gamma_{+}=\gamma_{-}$then any $C^{1}$ curve through a point of $\gamma_{+}$in either direction returns to $\alpha_{1}$ and since there are exàctly two switches on $\alpha_{1}$, actually crosses $\alpha_{1}$. This means every foliation carried by $\tau$ has a return leaf contradicting the definition of $\tau$. Thus $\gamma_{+}$comes after $\gamma_{-}$along $\gamma$. The branch preceding $\gamma_{+}$in the + direction is larger at the common switch since $\gamma_{+}$is the first branch to satisfy the given condition. Similarly the branch preceding $\gamma_{-}$is larger at the common switch. Either one of these two is a winged branch or there is one between them on $\gamma$ which is and we are done.

Suppose $\tau$ is type (I) carrying foliations with returning leaves to both sides of $\alpha_{1}$. As we have seen, this means there are arcs $\rho_{1}, \rho_{2}$ from $\alpha_{1}$ to switches $p_{1}, p_{2}$ and loops $\gamma_{1}, \gamma_{2}$ based at $p_{1}, p_{2}$ respectively. The loops $\gamma_{i}$ are disjoint from $\alpha_{1}$ and $\alpha_{2}$ and are not homotopic to $\alpha_{1}$. 
LEMMA 5.4. If $\tau$ is type (I), $\rho_{1}$ is disjoint from both $\rho_{2}$ and $\gamma_{2}$ and $\rho_{2}$ is disjoint from $\gamma_{1}$. If $\gamma_{1} \cap \gamma_{2} \neq \varnothing$ we can split the track along a branch of the intersection, giving new type (I) tracks for which $\gamma_{1} \cap \gamma_{2}=\varnothing$ as well.

Proof. We claim that there cannot be a branch on $\rho_{1}$ which is larger at a switch not on $\alpha_{1}$ but which is at the end of the branch closer to $\alpha_{1}$. If there were such a switch, consider a system of weights that puts much more weight on the branch $\bar{\rho}_{1}$ not on $\rho_{1}$ incident to the switch than on the smaller branch on $\rho_{1}$ and otherwise concentrates most weights along $\rho_{1}$. Any leaf from $\alpha_{1}$ that travels along $\rho_{1}$ past this switch and around $\gamma_{1}$ must follow $\bar{\rho}_{1}$ at the switch. This gives a foliation carried by $\tau$ without returning leaves to $\alpha_{1}$. The contradiction proves the claim. In particular, this means $\rho_{2}$ is disjoint from $\rho_{1}$. If $\rho_{2}$ has a branch in common with $\gamma_{1}$, there is a winged branch in $\rho_{2}$ larger at a switch not on $\alpha_{1}$ but at the end of the branch closer to $\alpha_{1}$ and we have the same contradiction as above with $\rho_{2}$ in place of $\rho_{1}$. Thus $\rho_{2}$ is disjoint from both $\rho_{1}$ and $\gamma_{1}$ and similarly $\rho_{1}$ and $\gamma_{2}$ are disjoint.

If $\gamma_{1} \cap \gamma_{2} \neq \varnothing$ they have a winged branch in common. Further, $\gamma_{1}$ and $\gamma_{2}$ do not cross for otherwise there would not be returning leaves to both sides of $\alpha_{1}$. Thus we can split the track along $\gamma_{1}$ and $\gamma_{2}$ so they become disjoint, proving the lemma.

Suppose now the track $\tau$ satisfies the conclusion of lemma 5.4. We form a new homotopy class $\alpha^{*}$ out of each $\gamma_{i}$. If $\gamma_{1}$ is not homotopic to $\alpha_{2}$, let $\alpha^{*}$ be $\gamma_{1}$. If $\gamma_{1}$ is homotopic to $\alpha_{2}$, let $\alpha^{*}$ be the simple closed curve homotopic to the curve found by following $\gamma_{1}$, then $\rho_{1}$, then $\alpha_{1}$ and then $\rho_{1}$ again. In either case, $\alpha^{*}$ has zero geometric intersection with both $\alpha_{1}$ and $\alpha_{2}$ and is homotopic to neither. We similarly construct the curve from $\gamma_{2}$; it has zero geometric intersection with $\alpha^{*}$, so it must be homotopic to $\alpha^{*}$. These two copies $\alpha_{1}^{*}, \alpha_{2}^{*}$ of the homotopy class of $\alpha^{*}$ bound an embedded open annulus $A$ by the disjointness of lemma 5.4.

LEMMA 5.5. There is either an embedding of $\alpha^{*}$ in $\tau$ with exactly two switches on $\alpha^{*}$ and one branch leaving on each side or $\tau$ is shift equivalent to such a track.

Proof. Consider the component of $\operatorname{Fr} A$ formed by $\alpha_{1}^{*}$. If $\alpha^{*}$ is homotopic to $\gamma_{1}$, there is a singularity on $\operatorname{Fr} A$ at the switch $p_{1}$. If $\alpha^{*}$ is homotopic to the union of curves, there is a singularity on $\operatorname{Fr} A$ on $\alpha_{1}$. In either case we have accounted for a singularity on the component of $\operatorname{Fr} A$ formed by $\alpha_{1}^{*}$. Similarly, there is a singularity on the other component. Since $\tau$ is complete, there must be an arc $\sigma$ in $A$ joining the two components so that all complements of $\tau$ are simply connected. Since both $\gamma_{i}$ and the path $\rho_{i}$ followed by $\gamma_{i}$ and then $\rho_{i}$ again are $C^{1}$ paths, there are two additional singularities from the joining of $\sigma$ to the two components of $F r A$ giving a total of four in this component. Since each component of the complement of $\tau$ must have three singularities, one of two possibilities must occur. Either there is a branch from $\sigma$ to itself creating two 3-gons as desired, or there is a second branch joining the boundaries creating two 3-gons. It is not difficult to see that any of the latter tracks is equivalent to one of the former. For example, the tracks in figure 16 are shift equivalent. This completes the proof of the lemma. 

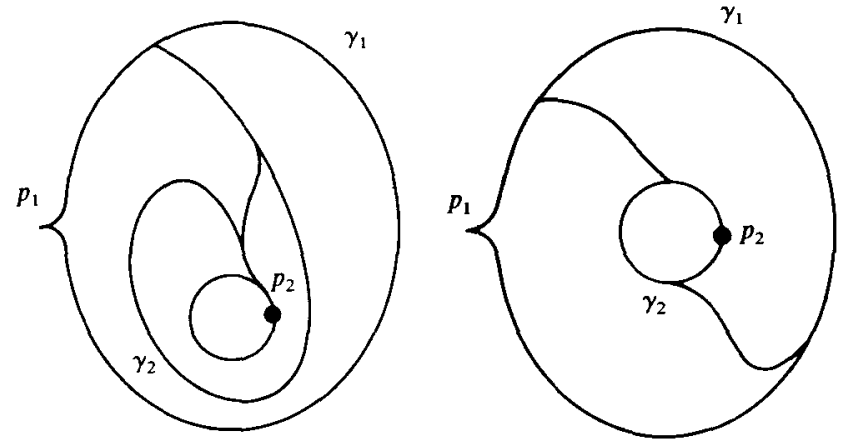

FIgURE 16

Lemmas 5.3 and 5.5 allow us to define a simplicial system to any minimal $F \in L$ using tracks of type (I) or (III). No $F \in L$ can be carried by a track type (II) since these are in the domain of discontinuity. Start with an admissible system $\bar{\alpha}$ and a track $\tau$ admissible for $\bar{\alpha}$ carrying a minimal $F \in L$. If $\tau$ is of type (I) split along a common branch of $\gamma_{1}$ and $\gamma_{2}$ if necessary so the conclusions of lemma 5.4 hold. Then replace $\alpha_{1}$ with $\alpha^{*}$ found in lemma 5.5. Now $\tau$ is of type (III) with respect to $\left(\alpha^{*}, \alpha_{2}\right)$. Now split the track away from the embedded curves so the track is either type (I) or (III). If the latter, split again; if the former, split if necessary along the loops, change the admissible system and continue. With this prescription the states of our simplicial process are tracks of type (I) and (III) and the symbols the sequence of projective linear maps $(*)$.

Now let $H_{i} \subset \Sigma, i=1, \ldots, n$, be polyhedra defined by the allowable weights on these tracks and $G_{i} \subset H_{i} \cap \mathcal{O}$ some open set which exists since $L \cup T$ has empty interior. After $n$ stages we have a track $\tau^{(n)}$ whose allowable weights form one of the $H_{i}$. If the weights of $F$ with respect to $\tau^{(n)}$ are $\lambda^{(n)}$, then the weights of $F$ with respect to $\tau$ are

$$
\lambda=\bar{A}_{n} \lambda^{(n)},
$$

where $A_{n}$ is the product of the $n$ matrices given by the splittings $(*)$. Thus $\bar{A}_{n}\left(H_{i}\right)$ parametrizes, with respect to weights on $\tau$, all foliations carried by $\tau^{(n)}$ and $\vec{A}_{n}\left(G_{i}\right)$ parametrizes foliations in $\mathcal{O}$ carried by $\tau^{(n)}$. The sets $\bar{A}_{n}\left(H_{i}\right)$ form the cover of $L$ by sets $V_{i}$ required in proposition 5.2 and the $\bar{A}_{n}\left(G_{i}\right)$ are the subsets $U_{i}$ in the hypothesis of that proposition.

Remark. Each time a track becomes type (I) and the admissible system is changed,

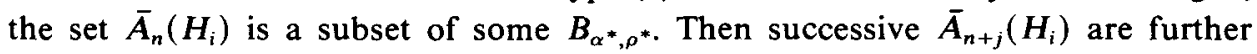
nested subsets of this $B_{\alpha^{*}, \rho^{*}}$ until a new system occurs.

We also note that this approach fails for $g \geq 3$ since the analogue of the lemmas is false. In particular there is not a number $k$ so that for any admissible $\alpha$, one of $k$ combinatorial tracks appears. This reflects the fact that the cover of theorem 3.6 is not disjoint.

To show that the hypotheses of proposition 5.2 are satisfied, we need only apply Kerckhoff's results [4] to our situation. We will recall a couple of concepts and definitions appearing in [4]. 
In the process of forming $A_{n+1}=A_{n} E_{1} E_{2}$ where $E_{1} E_{2}$ is the product of elementary matrices (*) we can think of $A_{n+1}$ as derived from $A_{n}$ by twice adding a column of $A_{n}$ to another column of $A_{n}$.

Consider a block of matrices in the expansion of some $F$. Take any subset of two or more columns and assume in this block each column in the subset has been added to some member of the subset and has had some member of the subset added to it. It is called isolated if no member has been added to a column outside the subset.

A simplicial process for which there is no non-trivial isolated collection of columns starting and ending with the same state is connected.

Proposition 5.5 (Kerckhoff). The simplicial process for a complete train track is connected.

Proposition 5.6 (Kerckhoff). For any connected simplicial process, for all $F$ except for a set of measure 0 , the columns of the matrices in the expansion of $F$ are $C$-distributed infinitely often for some $C>0$.

Proof of theorem 5.1. Suppose $m_{0}=m(L)>0$. Since the columns in the expansion of $F$ are $C$-distributed infinitely often, the ratio of the Jacobian of $\bar{A}_{n}$ is bounded at any two points infinitely often for a set of measure $m_{0}$. This means for the cover $\bar{A}_{n}\left(H_{i}\right)$ and $\bar{A}_{n}\left(G_{i}\right) \subset \bar{A}_{n}\left(H_{i}\right)$ the hypotheses of proposition 5.2 are satisfied and we have a contradiction.

\section{REFERENCES}

[1] A. Fathi, F. Laudenbach, V. Poenaru et al. Travaux de Thurston sur les surfaces, Asterisque, pp. 66-67 (1979).

[2] W. Floyd. Hyperbolic manifolds, 3 manifold constructions, and actions of subgroups of Mod $(\mathrm{g})$ on PL $\left(S_{g}\right)$. To appear.

[3] J. Harer \& R. Penner. Combinatorics of train tracks. To appear.

[4] S. Kerckhoff. Simplicial systems for interval exchange maps and measured foliations. Ergad. Th. \& Dynam. Sys. 5 (1985), 257-271.

[5] B. Maskit. Self-maps of Kleinian groups. Amer. J. Math 93 (1971), 840-856.

[6] H. Masur. Interval exchange transformations and measured foliations. Annals of Math. 115 (1982), 169-200.

[7] M. Rees. An alternative approach to the ergodic theory of measured foliations on surfaces. Ergod. Th. \& Dynam. Sys. 1 (1981), 461-485.

[8] W. Thurston. On the dynamics of diffeomorphisms of surfaces. Preprint.

[9] W. Thurston. The geometry and topology of 3-manifolds. Lecture Notes, Princeton.

[10] W. Veech. Interval exchange transformations. J. D' Analyse Math 33 (1978), 222-278. 